\title{
S3 Figure.
}

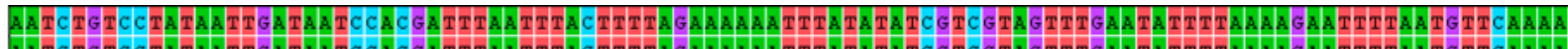
ATCTGTCCTATAATTGATAATCCACGATTTAATTTACTTTTA GAAAAATTTATATATCGTCGTA GTTT GAATATTTTAAAAGAATTTTAATGTTCAAA. AATCTGTCCTATAATTGATAATCCACGATTTAATTTACTTTTAGAAAAAATTTATATATCGTCGTAGTTTGAATATTTTAAAAGAATTTTAATGTTCAAAA ATCTGTCCTATAATTGATAATCCACGATTTAATTTACTTTTAGAAAAA TTTATATATCGTCGTAGTTTGAATATTTTAAAAGAATTTTAATGTTCAAA AATCTGTCCTATAATT GATAATCCACGATTTAATTTACTTTTAGAAAAAATTTATATATCGTCGTAGTTTGAATATTTTAAAA GAATTTTAATGTTCAAAA AATCTGTCCTATAATTGATAATCCACGATTIAATTTACTITTAGAAAAAATTATATATCGTCGTAGTTTGAATATTTTAAAAGAATITTAATGTTCAAAATT

$\frac{\mathscr{0}}{2}$ हั है

ATCTGTCCTATAATTGATAATCCACGATTTAATTTACTTTTAGAAAAAATTTATATATCGTCGTAGTTTGAATATTTTAAAAGATTTTAATGTTCAAAA 

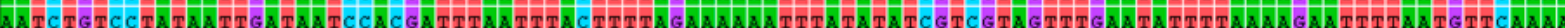

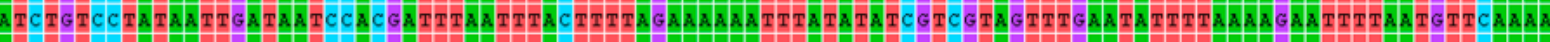
с a -

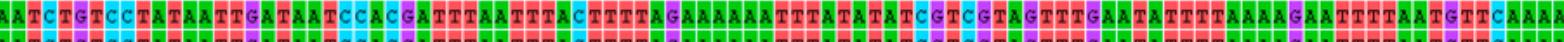

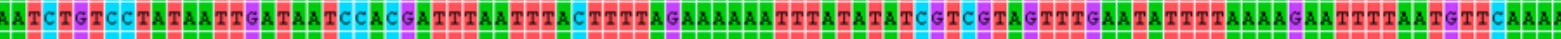

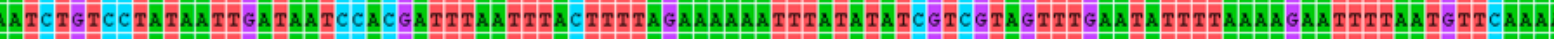

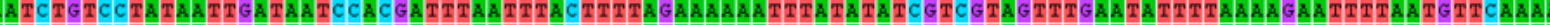
ATCTGTCCTATAATT GATAATCCACGATTTAATTTACTTTTAGAAAAAATTTATATATCGTCGTAGTTTGAA IA TIAAAGAALTTTAATGTTCAAA

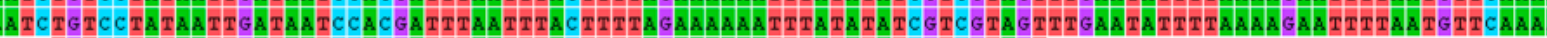

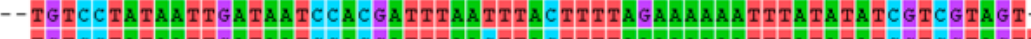

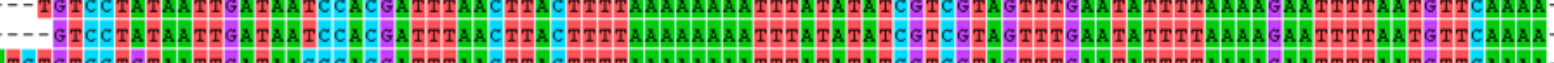

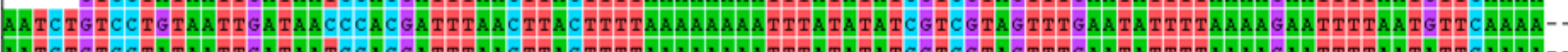

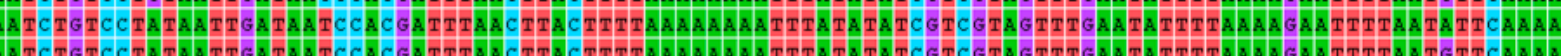

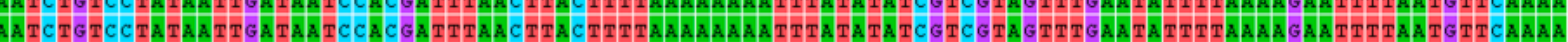

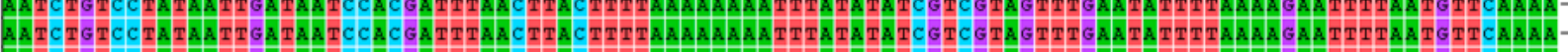

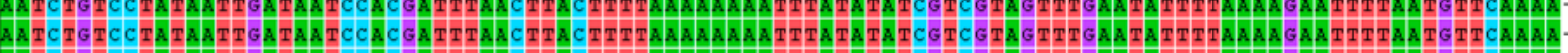

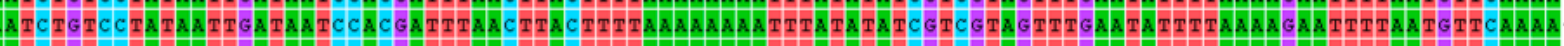

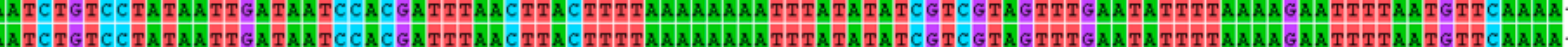

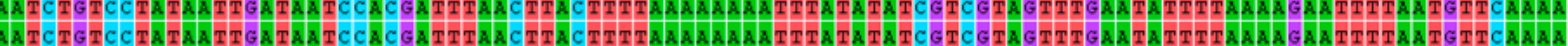

TTTTTTTATGAAATTAAATCAGATCAAGGTGTAGTTTATATAAA TT TIIITA GAATTAAA TCA GATCAAGGTGTAGTTTATATAAA

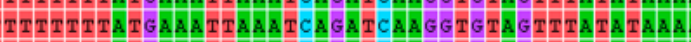
TTTTTTTATGAaATTAAATCAGATCAAGgTGTAGTTTATATAAA

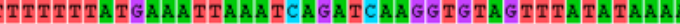
TTTTTTIATGAAATTAAATCA Ga TCAAGGTGTAGTTTATATAAA

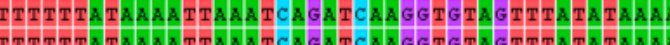

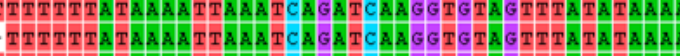

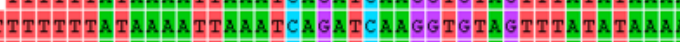

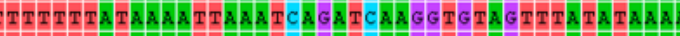
TTITTTTATAAAATTAAATCAGATCAAGGTGTAGTTTATATAAA

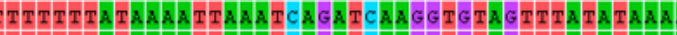

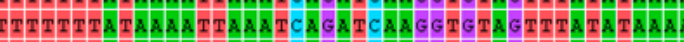
TTTTTTIATAAAATTAAATCAGATCAAGGTGTA GTTTATATAAA TITTTATAAA TTAAATCAGATCAAGGTGTAGTTTATATAAA

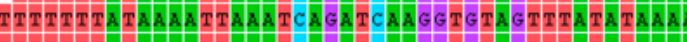
TITRT TATAAAATTAAATCAGATCAAGGTGTAGTITATATAAA

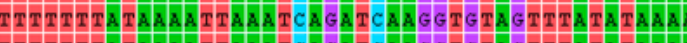

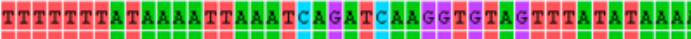
TTTTTTTA AA A A TTAAATCA GATCAA G GTGTA GTTTATATAAA.

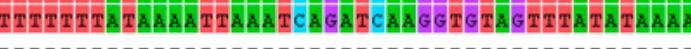
TTTTTTATAAAATTAARTCAGATCAAGGTGTAGTTTATA- -

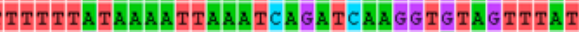

TTTTTTGTAAAATTAAATCA GA TCAA GGTATA GTTTATATAAA TITTTATAABATTABATCA GA TCAAGGTGTAGTTTATATAAA

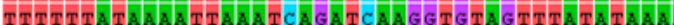
T. c -

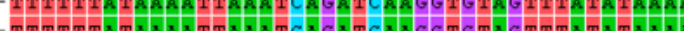
re TTTTTIATAAAATTAAATCAGATCAAGGTGTAGTTTATATAAA 